\title{
Severe Lower Gastrointestinal Bleeding in Crohn's Disease: Successful Control With Infliximab
}

Jacques Belaiche, M.D.; Edouard Louis, M.D.

Department of Gastroenterology, CHU Liège, Belgium

\section{TO THE EDITOR}

Acute lower GI bleeding is an uncommon but potentially life-threatening complication of Crohn's disease (CD) occurring in about $1.5 \%$ of cases $(1,2)$. The severity is judged on the basis of the need for transfusion of several units of blood. It represents a therapeutic challenge. A conservative approach has generally been advocated in view of the likelihood of spontaneous cessation of the bleeding. However, in some cases, particularly because of the risk of recurrence and multiple or diffuse bleeding lesions not allowing endoscopic treatment, there is a place for a drug with rapid mucosal healing potential (3-5). We describe two patients with severe bleeding caused by CD treated with infliximab.

The first patient was a 28-yr-old white woman with a 3-yr history of CD involving the terminal ileum and the colon who was admitted for massive rectal bleeding. She also suffered from recurrent perineal fistula. Two days before admission, she developed profuse rectal bleeding. On admission, the blood count showed a hemoglobin of $7.6 \mathrm{~g} / \mathrm{dl}$. Bleeding originating from upper GI tract or anal lesions was excluded. Colonoscopy showed a diffuse colonic involvement with multiple deep round or serpiginous ulcers located particulary in the cecum and me left colon. The colon contained a significant amount of blood, but the source of bleeding was not localized. After transfusion of two units of blood, oral budesonide $(9 \mathrm{mg}$ /day) and azathioprine $(125 \mathrm{mg} /$ day $)$ were started. Because the patient continued to bleed, requiring three more units of blood and because of persistent fistula discharge, a treatment with infliximab infusion $(5 \mathrm{mg} / \mathrm{kg}$ ) was started. The patient left the hospital without recurrence of bleeding 4 days after the first infusion. She was readmitted 10 days later for a rebleeding and a hemoglobin of $6.1 \mathrm{~g} / \mathrm{dl}$. A proctosigmoidoscopy showed circumferential ulcers and copious amounts of blood and melena without identifying an obvious site of bleeding. After transfusion of five units of blood, a second infusion of infliximab $(5 \mathrm{mg} / \mathrm{kg}), 14$ days after the first one, was administered. The patient was discharged without bleeding recurrence. A third infusion was administered 1 month later. At this time, there was no bleeding recurrence, and proctosigmoidoscopy revealed a complete healing of colonie ulcerations. The patient remains disease free without evidence of bleeding 5 months later.

The second patient was a 59-yr-old white woman with a 9-yr history of CD involving the colon who was admitted for rectal bleeding, diarrhea, and anemia. One month before admission, she noted increased diarrhea and frequent red blood per rectum. Medication on admission included oral steroids, metronidazole, and ciprofloxacine. On admission, the blood count showed a hemoglobin of $8 \mathrm{~g} / \mathrm{dl}$. Upper GI endoscopy was normal. Colonoscopy showed multiple deep round and large ulcers located in the right colon with blood ta the lumen, but no site of bleeding was identified. The rectum was normal. After transfusion of four units of blood, azathioprine $(100 \mathrm{mg} /$ day $)$ was started and first infusion of infliximab $(5 \mathrm{mg} / \mathrm{kg})$ was given. Diarrhea decreased, and rectorragia disappeared 4 days after infusion. Four months later, the patient had a relapse of CD but without evident bleeding. She was successfuly treated with a new infusion of infliximab.

The two cases show efficacy of infliximab in hemorragic forms of CD. This treatment allowed not only to control bleeding but also to scar underlying lesions. The primary treatment of severe lower GI bleeding in CD is determined by the severity and persistence of bleeding as well as the risk for recurrence. Localization of the site of bleeding is essential whether endoscopie therapy, surgery, or medical management is attempted. Endoscopie evaluation is preferable, if feasible, as it will provide a broader assessment of extent of disease as well as having a reasonable likelihood of identifying the site of bleeding with possible therapeutic intervention (2). If the acute bleeding ceases, an interval to attempt pharmacological therapy may be available, permitting treatment of the underlying CD. In our first case, severe acute bleeding resolved in two successive occasions a few days after infliximab infusion. This result may still be fortuitous. However, the fact that it occurred twice and the complete scarring of the lesion that was obtained later are arguments for a direct effect of infliximab, both in the early control of bleeding and in the absence of further recurrence. In our second case, mild-to-moderate bleeding had 
lasted for more than 1 month. It stopped within 4 days after infliximab infusion and did not recur. Again, such rapid effect after a longstanding bleeding is an argument for a specific role of infliximab.

In conclusion, our two cases show that new therapies such as infliximab, which may produce relatively rapid mucosal healing, may be beneficial and offer treatment alternative to surgery in either acute massive or chronic refractory bleeding in $\mathrm{CD}$.

\section{REFERENCES}

1. Pardi DS, Loftus EV, Tremaine WJ, et al. Acute major gastrointestinal hemorrhage in inflammatory bowel disease. Gastrointest Endosc 1999;49:153-7.

2. Belaiche J, Louis E, D'Heans G, et al. Acute lower gastrointestinal bleeding in Crohn's disease: Characteristics of a unique series of 34 patients. Am J Gastroenterol 1999;94:2177-81.

3. Cohen RD, Tsang JF, Hanauer SB. Infliximab in Crohn's disease: First anniversary clinical experience. Am J Gastroenterol 2000;95:3469-77.

4. Farrell RJ, Shah SA, Lodhavia PJ, et al. Clinical experience with infliximab therapy in 100 patients with Crohn's disease. Am J Gastroenterol 2000;95:3490-7.

5. van Dullem HM, van Deventer SJ, Hommes DW, et al. Treatment of Crohn's disease with anti-tumor necrosis factor chimeric monoclonal antibody (cA2). Gastroenterology 1995;109: 129-35. 